\title{
DIFFERENTIATED MANAGEMENT OF DEPRESSIVE DISORDERS IN ONCOLOGY PATHOLOGY
}

\author{
I. Marinescu ${ }^{1}$, D. Marinescu ${ }^{1}$, R.A. Schenker ${ }^{2}$, D.G. Malaescu ${ }^{3}$, P.O. Stovicek ${ }^{4}$ \\ ${ }^{1}$ University of Medicine and Pharmacy of Craiova, Department of Psychiatry, Craiova, Romania \\ ${ }^{2}$ University of Medicine and Pharmacy of Craiova, Doctoral School, Craiova, Romania \\ 3"Titu Maiorescu" University- Bucharest, Department of Morphopathology, Craiova, Romania \\ 4"Titu Maiorescu" University- Bucharest, Department of Pharmacology, Craiova, Romania
}

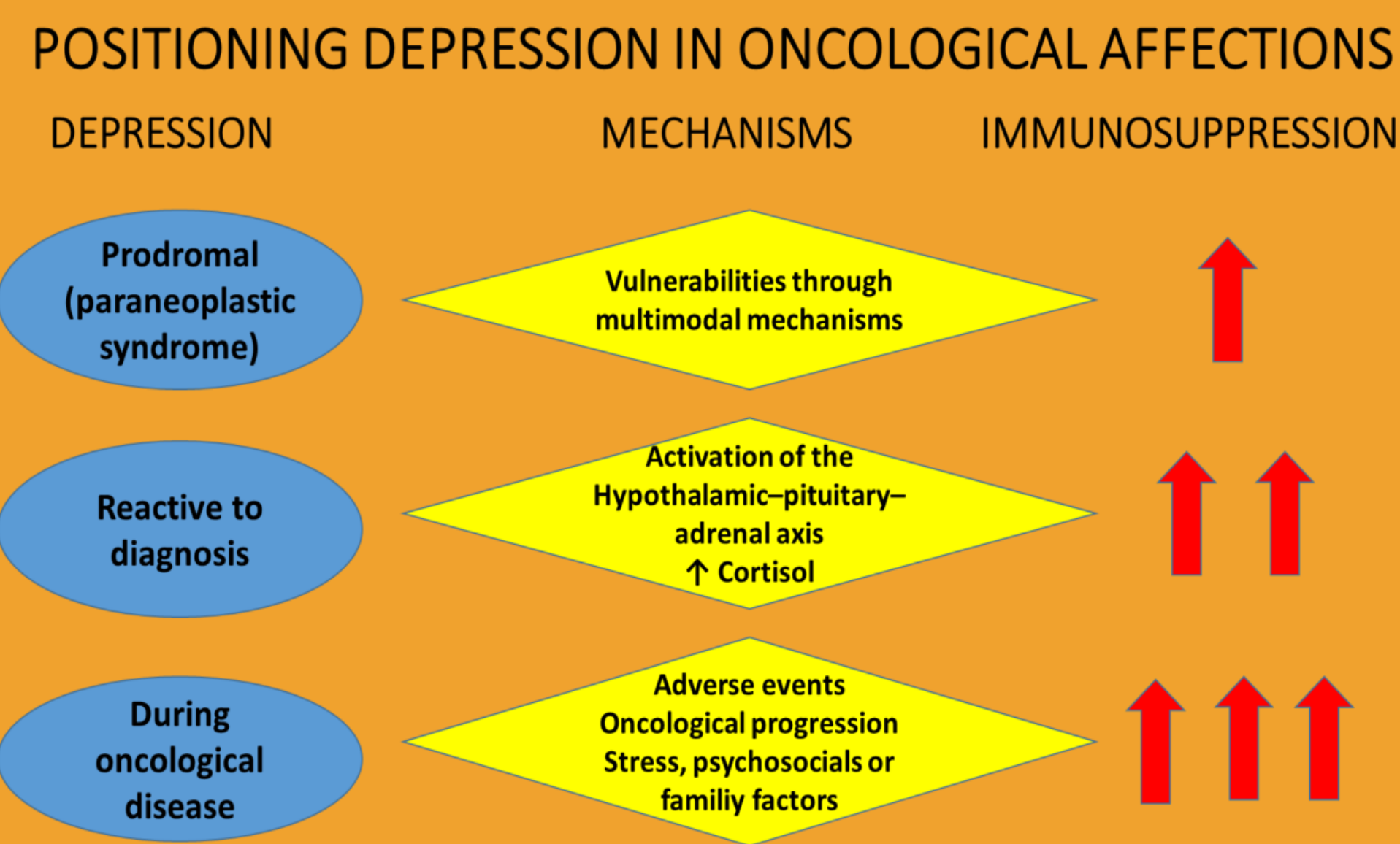

Figure 1. Types of depression in oncological affections and its mechanisms of action

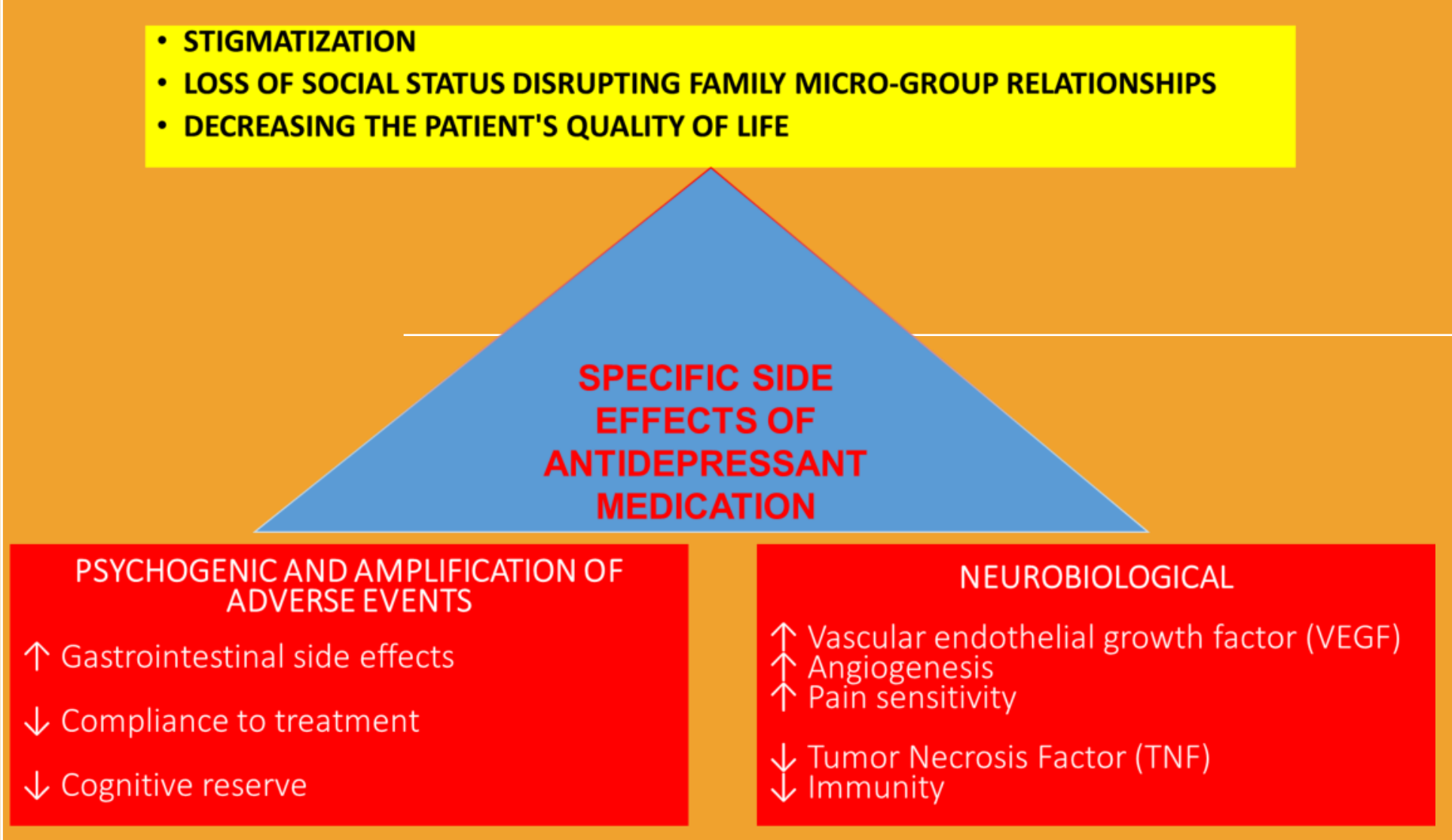

Figure 2. Specific side effects of antidepressant medicationion

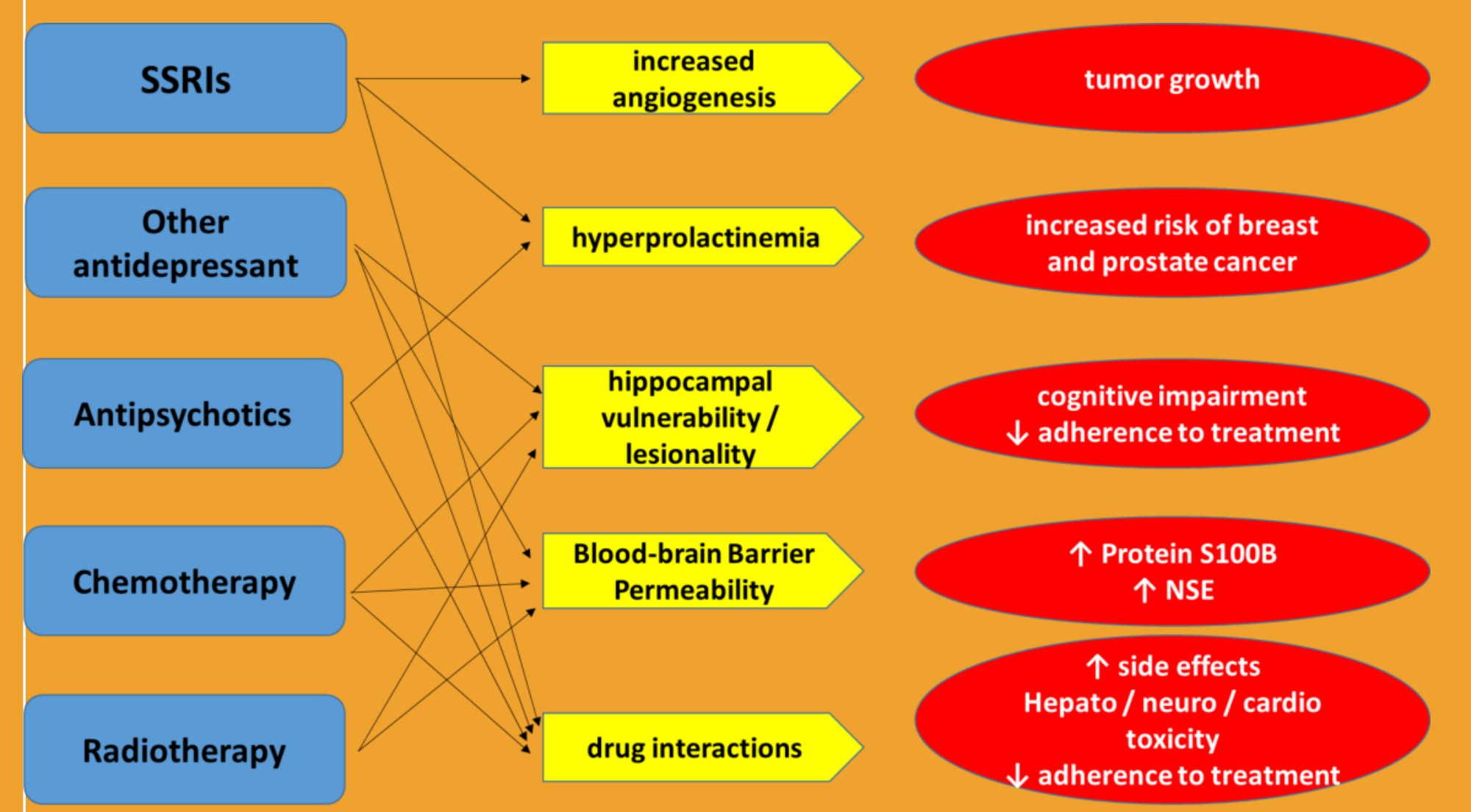

\section{Introduction:}

Epidemiological data prove depression as the most frequent psychiatric disorder associated with oncological pathology.

Objectives:

We believe that depression in oncology can be split in three clinical categories.

\section{Methods:}

The correct identification of these depression types (figure 1) and adopting differentiated strategies may improve the prognosis and the life quality of the oncology patient with depression.

\section{Results:}

1. In the prodromal phase, which we interpret as a real paraneoplazic syndrome (1). It precedes the oncologic disease by 5 years, correlated with the multi-systemic neurobiologic depression model and with moderate symptomatology (Hamilton scale <23), insomia, tiredness, algic elements frequently interpreted as fibromyalgia. Antidepressants associated or not with antipsychotic medication determines negative oncologic evolution (figure 2 ) through 2 mechanisms: A. Incomplete terapeutic response, with persistent symptomatology associated with biologic markers (increase of reactive $\mathrm{C}$ protein levels, 6 and 8 interleukins).

B. Consecutive advers events mechanisms induced by psychotropic medication intensifying inflamatory mechanisms and ARN markers modification mechanisms. Paraneoplazic depression requires multidisciplinary evaluation, associating depression markers with oncologic ones, for early diagnosys.

2. Associated with the disease, consecutive to oncologic diagnosis, under psychotraumatic and distress conditions, decreasing the patient's life quality and associating an avoidance behavior that delays treatment compliance. Evaluation and treatment require a psychoterapeutic team with psycho-oncologic competence (2).

3. Consecutive to neurotoxic side-effects of medication (3) (figure 3), with hippocampal atrophy, leading to secondary therapeutic rezistance, associated with the increase of 6 and 8 interleukins (progression factors of angiogenesis).

\section{Conclusions:}

Immune and inflammatory markers regards depression as an independent risk factor leading to unfavorably evolution. Consequently the customized treatment must consider such symptoms.

\section{References:}

1. Lorraine C. Pelosof, David E. Gerber, Paraneoplastic Syndromes: An Approach to Diagnosis and Treatment, Mayo Clin Proc. 2010;85(9):838-854.

2. Reema D. Mehta, Andrew J. Roth, Psychiatric considerations in the oncology setting, CA CANCER J CLIN 2015;65:299-314, doi: 10.3322/caac. 21285 .

3. Miyoung Yang, Changjong Moon, Neurotoxicity of cancer chemotherapy, Neural Regen Res. 2013 Jun 15; 8(17): 1606-1614. doi: 10.3969/j.issn.1673-5374.2013.17.009.

Figure 3. Side effects of antidepressant and oncological medications 